\title{
AN X-RAY BPM AND ACCOMPANYING ELECTRONICS
}

\author{
S. R. Marques", O. R. Bagnato, M. J. Ferreira, F. O. Bombacini, H. J. Onisto, LNLS, Campinas, \\ Brazil
}

\begin{abstract}
Recent experiments at the Brazilian Synchrotron Ligth Source (LNLS) Ultra Violet and X-Ray beamlines are pushing on the Synchrotron Radiation (SR) beam position stability requirements. In the direction of having photon Beam Position Monitor integrated to the orbit closed loop control system, we developed a staggered blades XR BPM and a four-channel current measurement electronics. The BPM mechanical design was based on the SR masks previously developed and currently in use at the beamline front ends. By this design reuse, in addition to using a readily-available, well-tested and low cost construction technique, we expect to have a simple replacement of the SR masks by the XR BPMs in most of the beamlines, shortening the downtimes and the number of line parts to be replaced. We describe the design and the resulting performance of the XR BPM and the accompanying electronics.
\end{abstract}

\section{INTRODUCTION}

The slow orbit feedback at the LNLS storage ring is based on the RF stripline BPMs and it has been improved as a result of efforts of the LNLS technical staff [1] [2]. Besides improving the existing system, we started considering the inclusion of XR BPMs in the orbit closedloop control system. Recently we bought a commercial XR BPM. The monitor head was acquired from FMB (Berlin, Germany), which also supplied the LCAD-4 electronics (Frank Optics). At the same time we developed a homemade version of an XR monitor and its electronics. The two complete systems were installed in 2004 at the LNLS diagnostics dedicated beamline.

The main mechanical part of the XR BPM is the staggered pair blades, which is positioned so that they intercept only the edges of the photon beam vertical distribution. The four photocurrent-generated signals are measured by the electronics and the center of gravity of the XR beam can be calculated from the rate (difference over sum) of these currents [3] [4].

\section{XR BPM MECHANICAL DESIGN}

One of the interesting features of the monitor head is that it was designed to replace the SR masks currently in use at the LNLS beamlines in such a way that its future installation in any beamline will not be a very complicated and time consuming task.

The monitor was built of high purity copper and brazed at the stainless steel flanges, in order to guarantee a good dimensional accuracy. The blades are isolated from each other and from the mask by thin alumina plates, which

\#sergio@lnls.br have good thermal conductivity and high electrical isolation. The screws are isolated from the blades by Teflon tubes.

Figure 1 illustrates the monitor mechanical design.

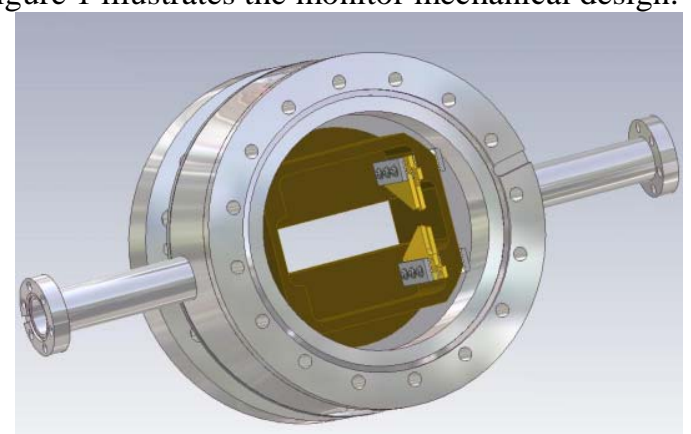

Figure 1: Mechanical design of the LNLS XR BPM. The water input and output connectors between the flanges are not visible.

Figure 2 shows the LNLS XR BPM with the blades and other internal parts not mounted.

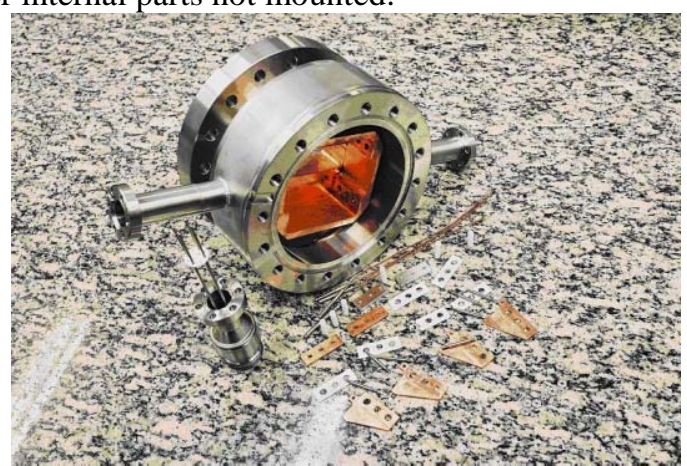

Figure 2: LNLS XR BPM. It is possible to see the electrical feed-through, the blades and the isolating plates and tubes. The mask is mounted in a CF100 flange, the internal aperture for the beam is about $20 \mathrm{~mm} \times 74 \mathrm{~mm}$ and the blades are about $25 \mathrm{~mm}$ decentralized. As shown in figures 1 and 2, the electrical connector can be installed in two positions, in order to facilitate the installation in different front ends.

\section{XR BPM ELECTRONICS}

Besides the dedicated electronics, good doublescreened cables are necessary for providing high electrical insulation and not decreasing the signal quality in the way from the monitor to the electronics. We used Huber+Suhner low noise triaxial cables to conduct the low photocurrent signal. The connector adopted at the monitor's head end is a four-pole Amphenol circular connector. At the electronics end we used triaxial connectors from Trompeter Electronics.

The first prototype of the XR BPM electronics, called MPRX IV, was designed to be completely analog and to 
occupy only one printed circuit board (PCB). Figure 3 shows the simplified block diagram of the MPRX IV and the figure 4 shows a picture of the MPRX IV prototype.

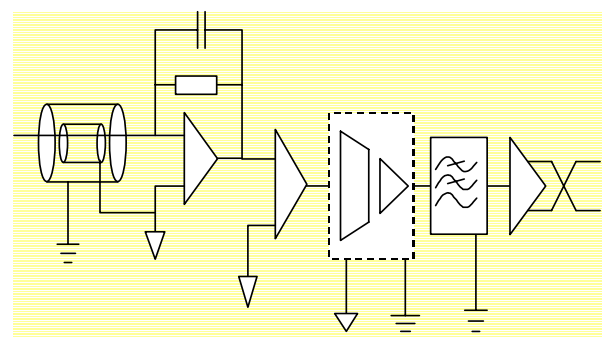

Figure 3: Simplified block diagram of the MPRX IV. Only one of the four channels is shown.

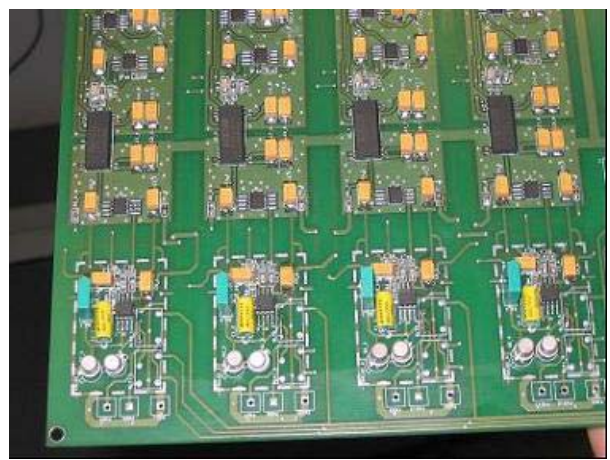

Figure 4: Picture of the four analog channels of the MPRX IV board. DC power supplies and the Bias voltage power supply are not shown.

In figure 3, the $\mathrm{RC}$ circuit determines the transimpedance gain and the bandwidth of the first stage, which are about $216.500 \mathrm{Volt} / \mathrm{Ampere}$ and $50 \mathrm{~Hz}(3 \mathrm{~dB})$, respectively. Note that the stability of these components is crucial for the proper operation of the system, since different drifts in the channels will cause a false photon beam position change that cannot be compensated. In the same way, the leakage in the filter capacitors cannot change. We used series Z201 $0.01 \% 1 \mathrm{ppm} /{ }^{\circ} \mathrm{C}$ low drift precision Vishay resistors and high quality polycarbonate capacitors from RTI Electronics. The operational amplifiers used in this circuit are the very low input bias current OPA129UA, from Texas Instruments. The gain spread among the four current to voltage converters is $0.011 \%$.

The next stage only applies a voltage gain of two. The component chosen was the INA137UA, also from Texas Instruments. These audio receivers have laser-trimmed internal resistors, which guarantee a good repeatability of the gain. The gain spread of the first two stages among the four channels is $0.016 \%$. At this point the voltage noise observed is comparable with ambient noise measured by the scope (Tektronix 684B). No special techniques for noise level measuring were adopted. The gain and offset characterization was done with an Agilent $7 \frac{1}{2}$ digit multimeter model 34720A.

Note that there are two potentials in the circuit: the bias voltage, at the monitor side, and the ground, at the output signal side. Biasing the blades is necessary to avoid space charge effects and cross talk, which degrades the measurement resolution. The isolator amplifier used to take the signal to the ground potential is the ISO124UA, from Texas Instruments. These amplifiers use a duty cycle modulation-demodulation technique that decreases the noise performance and the gain repeatability among the channels. The gain spread among the four channels measured at the isolator's outputs is $0.15 \%$.

The $50 \mathrm{~Hz}$ output filter was implemented with an OPA227UA (Texas Instruments) in a Salen Key - Bessel configuration. A DRV135UA audio differential line driver from Texas Instruments provides the output signal, and it improves the output signal transmission noise immunity.

Figure 5 shows a bench test where it is possible to observe the noise performance and the stability of the MPRX IV during about 24 hours.

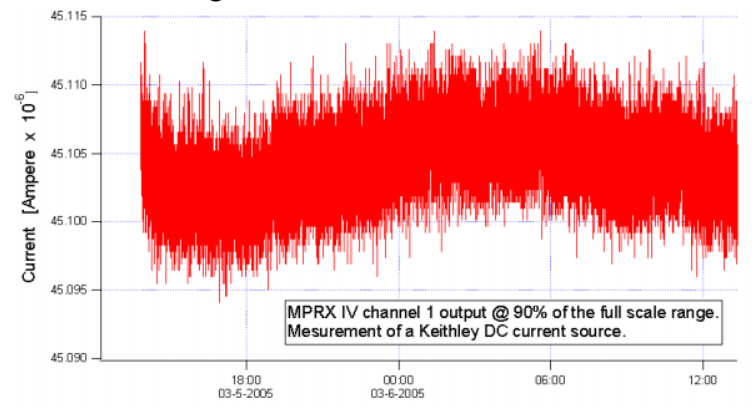

Figure 5: Bench test realized with the MPRX IV. The four channels demonstrated similar behaviors.

For the test shown in figure 5, we used a "model 230" Keithley precise voltage source and a set of Vishay precision resistors to implement the current source. The output data was taken from our dedicated 16 bits ADC cards, whose resolution is at least one order of magnitude better than the data obtained. After this analog to digital conversion, the equivalent current noise observed is less than $20 \mathrm{nA}$ pick-to-pick. Note that for the MPRX IV, $1 \mathrm{mV}$ corresponds to about $4.5 \mathrm{nA}$.

Besides the noise, the linearity at low-level measurements determines the sensitivity of the board. Non-linearity effects are commonly caused by current leakages due the substrate material, package design and the PCB layout. Active components that are not suitable for low-level measurements can also decrease the performance. We used a "model 225" Keithley Current Source to perform the test. Figure 6 shows the linearity test result.

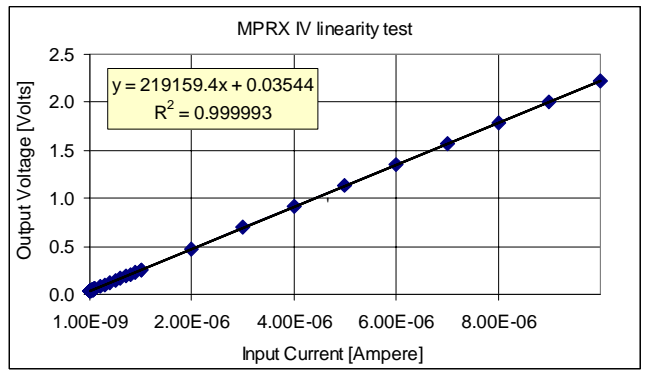

Figure 6: Linearity test realized with the MPRX IV. The result of one channel is shown. 
Through the figure 6 is not possible to observe any noticeable non-linearity effect above $1 \mathrm{nA}$. But, if instead of making one linear fit for the whole data, we separate groups of points between $1 \mathrm{nA}$ to $10 \mathrm{nA}, 10 \mathrm{nA}$ to 100 $\mathrm{nA}$, and so on up to $10 \mu \mathrm{A}$, we will find a $2 \%$ gain change for the data bellow $10 \mathrm{nA}$ compared to the remaining data. Besides that, the gain spread among the channel increase from less than $0.2 \%$ to almost $4 \%$. These observations probably show us the initial effects of some non-linearity behavior.

We intend to perform more measurements to better understand the non-linearity effects and to determine more precisely the low current limit of the MPRX IV board. However the photo-currents at the diagnostics beamline are larger than 10 micro Ampere, so that these tests were not necessary to put the XR BPM in operation.

\section{PRACTICAL CONSIDERATIONS}

Besides the gain spread among the four channels of the MPRX IV, we measured an offset spread of about $190 \mathrm{nA}$ (equivalent current) among the four channels, mainly due to the isolator. The temperature drifts of these parameters are small: the worst performance component in the chain (the isolator) should change $\pm 10 \mathrm{ppm} /{ }^{\circ} \mathrm{C}$ and the offset should change $200 \mu \mathrm{V} /{ }^{\circ} \mathrm{C}$.

The design of the MPRX IV was done in a such way that there is no analog calibration capability on the PCB. Similar gains and offset values would be better, but the calibration could be done by software and the high performance components chosen for the design have temperature drift specifications much better than those of the discrete components that are commonly used to perform offset nulling and gain calibration.

\section{PHOTON BEAM MEASUREMENTS}

We have performed beam position measurements at the diagnostics beamline and figure 7 shows the data of the two monitors: the commercial and the homemade ones.

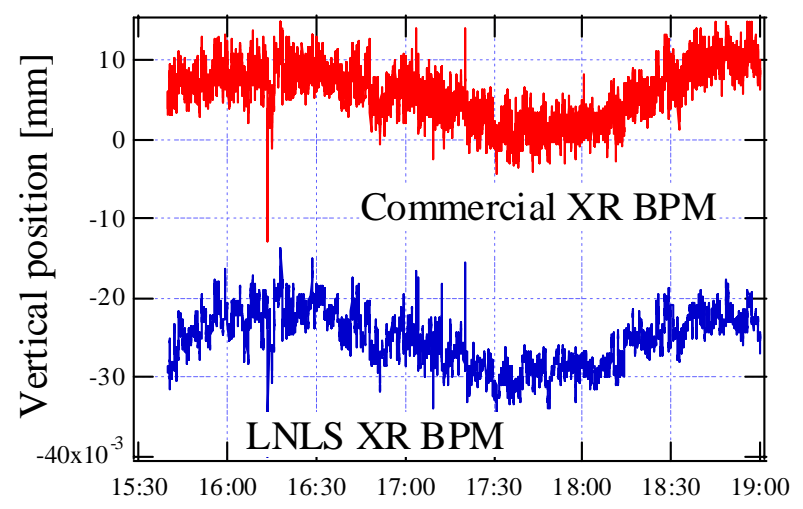

measurements in the diagnostics beamline. Data of the two XR monitors are in accordance.

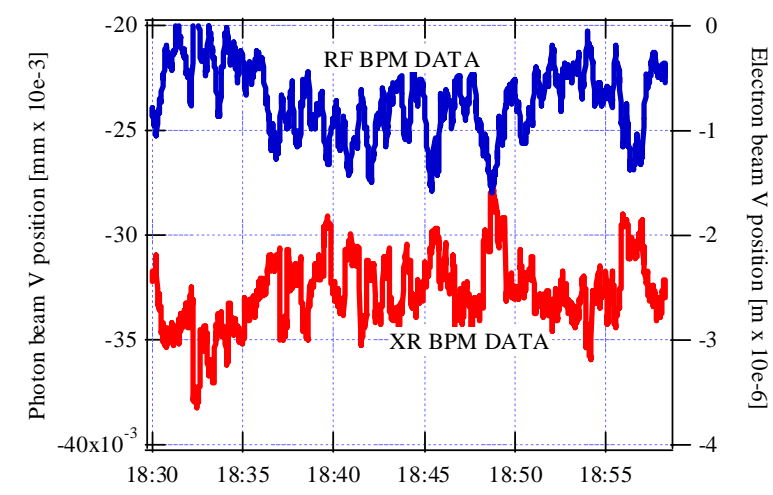

Figure 8: Correlation observed between the data of the RF BPM just after the dipole 02 and the data of the homemade XR BPM at the diagnostics beamline (in the same dipole).

\section{CONCLUSIONS}

The XR BPM has been tested in the diagnostics beamline and the results are in agreement with the commercial XR BPM. It is possible to correlate various electron beam movements with the XR BPM data.

The low limit measurement capability is related to noise and non-linearity problems. We are working on a new version of a MPRX IV and we hope to reduce considerably these limiting factors. We hope that in the near future we can routinely cross check the RF BPMs readouts using the XR BPM data. The experiments to include the XR BPM in the orbit closed-loop control system are still at the beginning, and it will probably take some months to decide whether to include it or not in the slow orbit feedback system.

\section{ACKNOWLEDGEMENTS}

The authors are in debt with technical staff of the LNLS for the help with the XR BPM construction and installation. We also want to acknowledge Texas Instruments for the samples program, which allowed fast prototyping and evaluation.

\section{REFERENCES}

[1] L. Lin, "The Brazilian Light Source”, IBWS2004, Grindelwald, Switzerland, December 2004, http://iwbs2004.web.psi.ch/documents/program/Liu. Lin/1_compact.pdf.

[2] L. Lin, "Orbit Stability at the Brazilian Synchrotron Light Source", these proceedings.

[3] K. Holldack, Private communication.

[4] W. B. Peatman, K. Holldack, "Diagnostic front end for BESSY II", Journal of Synchrotron Radiation, Volume 5, Part 3, Pages 639-641, May 1998. 